\section{Histoire Épistémologie Langage}

43-2| 2021

Linguistique psychologique et sémiotique : le contexte allemand et son influence

\title{
L'apport de la linguistique psychologique allemande à la psychologisation des sciences humaines russes. Le cas de la syntaxe psychologique
}

\section{Sergueï Tchougounnikov}

\section{OpenEdition Journals}

Édition électronique

URL : https://journals.openedition.org/hel/924

DOI : $10.4000 /$ hel.924

ISSN : $1638-1580$

Éditeur

Société d'histoire et d'épistémologie des sciences du langage

Édition imprimée

Date de publication : 31 décembre 2021

Pagination : 31-56

ISBN : 9791091587150

ISSN : 0750-8069

\section{Référence électronique}

Sergueï Tchougounnikov, « L'apport de la linguistique psychologique allemande à la psychologisation des sciences humaines russes. Le cas de la syntaxe psychologique ", Histoire Épistémologie Langage [En ligne], 43-2 | 2021, mis en ligne le 01 décembre 2021, consulté le 17 décembre 2021. URL : http:// journals.openedition.org/hel/924 ; DOI : https://doi.org/10.4000/hel.924

\section{(c)}

HEL is licensed under a Creative Commons Attribution-NonCommercial-NoDerivatives 4.0 International License 


\title{
L'APPORT DE LA LINGUISTIQUE PSYCHOLOGIQUE ALLEMANDE À LA PSYCHOLOGISATION DES SCIENCES HUMAINES RUSSES LE CAS DE LA SYNTAXE PSYCHOLOGIQUE
}

\author{
Sergueï Tchougounnikov
}

Université de Bourgogne Franche-Comté, Dijon, France

\begin{abstract}
Résumé - Entre 1860 et 1930, sous l'impulsion des recherches allemandes issues du courant dit de la « linguistique psychologique », notamment à travers les travaux de H. Steinthal (1823-1899), G. von der Gabelentz (1807-1874) et H. Paul (1846-1921), la linguistique russe connait un vaste mouvement de psychologisation qui se manifeste en particulier dans le domaine de la syntaxe. Cette étude dresse un aperçu de différentes théories de la syntaxe psychologique développées par les linguistes russes au cours de cette période. On y mènera l'analyse des concepts de «syntaxe évolutionniste» (A. Potebnia, 1835-1891); de «syntaxe énergétique » (D. Ovsianiko-Koulikovski, 1853-1920); de «syntaxe formaliste » (F. Fortounatov, 1848-1914); de «syntaxe volontariste » (A. Pechkovski [Peškovskij], 1878-1933); enfin, de «syntaxe communicative » (A. Chakhmatov [Šakhmatov], 1864-1920).
\end{abstract}

Mots-clés - linguistique psychologique, syntaxe psychologique, représentation, conscience verbale, histoire de la linguistique allemande, histoire de la linguistique russe
Abstract - This study deals with the conceptualisation of psychological syntax set forth in Russia from 1860 to 1930 under the influence of the German psychological linguistics, particularly in the works of H. Steinthal (1823-1899), G. von der Gabelentz (1807-1874) and H. Paul (18461921). This overview of the positions of Russian linguists on psychological syntax involves the analysis of the "evolutionary syntax" of A. Potebnja (1835-1891); the "energetic syntax" of D. Ovsianiko-Kulikovsky; the "formalist syntax" of F. Fortunatov (1848-1914); the "voluntarist syntax" of A. Peshkovsky [Peškovskij] (18781933); and the "communicative syntax" of A. Shakhmatov [Šakhmatov] (1864-1920).

Keywords - psychological linguistics, psychological syntax, representation, verbal consciousness, German linguistics, Russian linguistics

\section{INTRODUCTION}

Cette étude porte sur différentes théories de la syntaxe psychologique développées en Russie de 1860 à 1930 sous l'impulsion des recherches allemandes issues du courant dit de «la linguistique psychologique»(1850-1920). Les idées des linguistes russes en la matière ont en effet reçu l'influence directe des travaux d'un certain nombre de savants allemands ayant contribué à la promotion et la 
mise en pratique d'une syntaxe d'ordre psychologique, notamment Heymann Steinthal (1823-1899), Georg von der Gabelentz (1807-1874) et Hermann Paul (1846-1921). Steinthal entreprend cette psychologisation de la syntaxe dès son ouvrage de 1855 , où il pose le problème en termes plus généraux de différence entre grammaire et logique, phrase et jugement, langage et pensée, tout en cherchant à combattre « la prétendue indissociabilité et unité du langage et de la pensée » ${ }^{1}$. Gabelentz introduit les notions de «sujet psychologique» et de «prédicat psychologique » dans un article de 1869; il les reprend ensuite dans son étude de 1874 et dans sa monographie de $1891^{2}$.

Pour H. Paul, la syntaxe psychologique illustre l'une des idées maîtresses de ses Prinzipien der Sprachgeschichte (1880-1920), celle selon laquelle il existe une dichotomie naturelle des catégories grammaticales et psychologiques. Elles forment une hiérarchie « naturelle » : la catégorie psychologique est première par rapport à la catégorie grammaticale. Cette dernière apparait comme une sorte de formalisation d'une «prégnance» ou d'une «pertinence» psychologique antérieure ${ }^{3}$.

Cet aperçu des théories des linguistes russes relatives à la syntaxe psychologique s'attachera à l'analyse des concepts de "syntaxe évolutionniste", d'Aleksandr Potebnia (1835-1891); de «syntaxe énergétique», de Dmitri Ovsianiko-Koulikovski (1853-1920); de «syntaxe formaliste», de Filipp Fortounatov (1848-1914); de «syntaxe volontariste», d'Aleksandr Pechkovski [Peškovskij] (1878-1933); enfin, de «syntaxe communicative», d'Alekseï Chakhmatov [Šakhmatov] (1864-1920).

Nous avons retenu pour cet exposé les linguistes russes qui nous semblent les plus représentatifs au sein du courant qu'on appelle habituellement le « courant psychologique » ou encore la «linguistique psychologique» russe et dont les dates se situent approximativement entre 1860 et 1930 . La « linguistique psychologique » s'est développée initialement en Allemagne à partir des années 1850 et ensuite en Russie. Le cas russe est dans ce contexte particulièrement intéressant. Il fournit le lien conceptuel manquant à la généalogie de la tradition psycholinguistique actuelle. Ce statut quelque peu «exemplaire» s'explique par des circonstances historiques et culturelles singulières, en particulier par toute une tradition d'échanges culturels et scientifiques entre Allemagne et Russie ${ }^{4}$.

La linguistique allemande et la linguistique russe ont évolué, au cours du $\mathrm{XIX}^{\mathrm{e}}$ siècle, dans une sorte de remarquable et intense interaction. Au-delà d'un simple parallélisme ou d'un jeu d'influences, ces deux linguistiques ont pu formuler deux programmes de recherche convergents. Le courant psychologique,

1 Voir : « Présentation détaillée de la différence entre grammaire et logique », ainsi que les développements autour de la question : « Langage et pensée sont-ils identiques ? » dans Steinthal $1855: 152-153$, etc.

2 Les notions de « sujet psychologique » et de « prédicat psychologique » sont introduites dans « Ideen zu einer vergleichenden Syntax. Wort- und Satzstellung » [Idées pour une syntaxe comparée. La position du mot et de la phrase] (1869) ; puis, comme indiqué, rediscutées dans Gabelentz 1874 : 129-165 et Gabelentz 2016 [1891-1901] : 385-389.

3 Paul 1909 [1880] : 124-127.

4 Voir à ce sujet Romand et Tchougounnikov 2009 : 3-27. 
tel qu'il s'est manifesté dans ces deux pays, offre un très bon exemple de cette dynamique de transferts scientifiques. En effet, c'est précisément cette linguistique outillée de notions psychologiques qui, dans ces deux traditions respectives, en est venue à constituer, vers la fin $\mathrm{du} \mathrm{XIX}^{\mathrm{e}}$ siècle, un véritable programme de recherche national ${ }^{5}$.

Tous les linguistes russes importants du XIX ${ }^{\mathrm{e}}$ siècle sont passés par l'école allemande - soit par le biais de stages et de séjours scientifiques, soit par les lectures et par diverses affinités biographiques et langagières ${ }^{6}$. L'influence allemande sur la linguistique russe commence à se manifester dans les années 1840 et se traduit très vite par une psychologisation croissante des programmes de travail. À partir de ce moment, on assiste à une perte d'influence de la tradition linguistique française (notamment, du programme de la grammaire générale) et à la multiplication corrélative des références à la tradition linguistique allemande.

\section{APPROCHES DE LA SYNTAXE PSYCHOLOGIQUE DANS LE CONTEXTE RUSSE}

\subsection{Potebnia : la syntaxe évolutionniste}

Le linguiste Aleksandr Potebnia (1835-1891), professeur à l'Université de Kharkov, et que l'on désigne souvent comme le fondateur et chef de file de «l'école de Kharkov», se réclame ouvertement du courant de la « linguistique psychologique ». Ainsi, il fait remarquer :

Quand on dit que « le premier mot est déjà une proposition », on entend inconsciemment par «proposition» non pas le phénomène qui porte ce nom dans les langues formelles, mais un jugement psychologique (et non pas logique) à l'aide des mots. Ce premier mot [...] ou cette proposition primitive, ce qui est la même chose, est un acte d'aperception, c'est-à-dire de comparaison et d'explication de ce qui avait été perçu ; ce mot-proposition primitif relie deux entités mentales : ce qui est à expliquer (le sujet psychologique) et ce qui explique le premier (le prédicat psychologique); dans ce sens, ce mot-proposition est à deux termes, sans quoi ni la comparaison ni l'explication ne sont pensables. Mais le sujet de ce motproposition (une perception diffuse d'une image externe, accompagnée d'une sensation individuelle ou cette même sensation) est une question inconsciente qui n'est pas encore exprimée verbalement, et qui s'adresse à l'activité de la pensée à venir $[\ldots]^{7}$. (Potebnia $1958[1874]: 81-82$ )

Pour Potebnia, il n'y a que le prédicat (c'est-à-dire la représentation de l'élément explicatif) qui se trouve verbalement exprimé dans ces propositions primitives selon la formule : $\mathrm{X}$ (l'élément non exprimé verbalement et inconnu du locuteur jusqu'alors) = a (l'élément exprimé, par exemple : maman !). La proposition primitive à un terme ou le mot primitif de la langue, comme celui des

5 De même que, quelque temps auparavant, on a vu en Allemagne la linguistique historique et comparée se constituer en un programme de recherche national.

6 Pour ne citer que les noms les plus significatifs : Fiodor Bouslaev; Izmail Sreznevski ; Aleksandr Potebnia ; Dmitri Ovsianiko-Koulikovski ; F. Fortounatov ; J. Baudouin de Courtenay.

7 Toutes les traductions du russe sont les miennes. 
enfants qui commencent à parler, sont prédicatifs. Cette propriété continue à caractériser l'emploi des mots isolés tels que : Sjuda! (Ici !) ; Pora! (Il est temps !), etc. (ibid. : 82).

Les idées d'A. Potebnia relatives à la syntaxe psychologique sont entièrement dérivées de la conception aperceptive du psychisme formulée par Steinthal. Ce dernier assimile la structure syntaxique de base, celle de la phrase, au dispositif psychique de l'aperception; la phrase elle-même est perçue comme la conséquence et l'effet de ce mécanisme inhérent au psychisme ${ }^{8}$. Steinthal définit l'aperception comme « le mouvement de deux masses des représentations l'une à la rencontre de l'autre pour engendrer une connaissance $»^{9}$. Sa définition de l'aperception se fonde globalement sur celle de la psychologie herbartienne ${ }^{10}$.

Ainsi, dans le chapitre IX de son traité intitulé Langage et pensée, Potebnia reproduit fidèlement la définition de l'aperception par Steinthal, laquelle sera plus tard reprise par Paul dans ses Prinzipien. Potebnia écrit : "Ce qui est aperçu [apercipiruemoe], ce qui est à expliquer, forme le sujet du jugement. Ce qui est apercevant [apercipirujuščee], ce qui est déterminant, forme son prédicat. » Pour l'auteur, l'aperception est « le premier acte de la pensée », et le jugement est « la forme essentielle de la pensée ». La représentation (predstavlenie) est selon lui « le contenu déterminé de notre pensée » (Potebnia 1999 [1862] : 126).

La représentation n'est nullement significative en elle-même, elle l'est seulement en tant que forme, sous laquelle l'image sensible (čuvstvennyj obraz) entre dans la conscience. La représentation n'est possible que dans le mot ; le mot, pris isolément, hors combinaisons avec d'autres mots, exprime un jugement. Tout mot est, selon Potebnia, « une grandeur à deux éléments [dvučlennaja veličina] qui consiste en une image et sa représentation ». Dans les phrases nominatives à un mot $^{11}$, ce mot isolé peut s'expliquer de la façon suivante : cela (l'intuition sensible [čuvstvennoje vosprijatie] du vent) est ce (l'image sensible antérieure) que je perçois comme soufflant (la représentation de l'image sensible antérieure). La perception nouvellement « aperçue » sera sujet, et la représentation, qui est la seule à être exprimée par le mot, remplacera le vrai prédicat. Lors de la compréhension par le locuteur, la valeur des éléments du jugement sera inversée. Le son émis par l'autre provoquera chez l'interlocuteur le souvenir du même son

8 Sur l'«essence de l'aperception», voir Steinthal 1881 [1871]: 166-197; sur le fonctionnement « syntaxique » de l'aperception, ibid. : 198-206; sur le rôle de l'aperception dans la formation du mot et de la phrase ainsi que dans l'évolution du langage, ibid. : 396-427.

9 Steinthal 1881 [1871] : 171. L'aperception est surtout théorisée par Steinthal dans sa reprise de la partie psycholinguistique de son ouvrage de 1855, publié en 1871 sous le titre Abriss der Sprachwissenschaft, vol. 1 : Die Sprache im Allgemeinen. Einleitung in die Psychologie und Sprachwissenschaft.

10 Herbart appelle « aperception » le processus de fusion d'une nouvelle représentation avec la masse des représentations précédentes. La représentation ne peut devenir claire qu'en relation avec la totalité de l'expérience précédente. Si une nouvelle représentation ne trouve pas un tel appui, elle ne sera jamais « claire », elle n'accédera jamais à la conscience. Ainsi, l'existence même de nouvelles représentations est tributaire de l'interaction des perceptions passées et présentes (voir le chapitre $5:$ « Sur les effets conjugués [Zusammenwirken] de plusieurs masses [Vorstellungsmassen] de représentations » dans Herbart 1965 [1816] : 30-32 ; voir aussi le chapitre 6 : «Premier aperçu sur la liaison [Verbindung] entre l'âme et le corps », ibid. : 33-36).

11 Potebnia cite comme exemple l'exclamation Veter! (Vent!). 
qu'il avait jadis émis. Ce son permettra à la conscience de l'interlocuteur d'accéder à la « forme interne », c'est-à-dire, à la représentation, qu'il exprime. Enfin, à partir de là, la conscience accédera à l'image sensible de l'objet évoqué par ce son. Ainsi, le modèle de la compréhension par l'auditeur se présente comme suit : son - « forme interne » (représentation) - image sensible (ibid. : 126-127).

Potebnia définit, à l'instar de Steinthal, le prédicat psychologique comme l'élément essentiel de la phrase. Pour lui, la perception nouvellement apparue dans la conscience conduit à l'acte de l'aperception. Le locuteur "aperçoit», en prononçant un mot qui correspond à cette nouvelle perception. Cela faisant, il émet le mot qui possède la valeur d'un prédicat. En accentuant l'élément le plus important parmi ceux qui sont entrés en combinaison, le locuteur « anéantit par là l'indifférence originelle des éléments constitutifs de l'aperception ». Cet élément accentué est le prédicat dont le locuteur fait pour la seconde fois l'objet de sa pensée (ibid. : 134).

Chez Potebnia, les catégories du langage et surtout les parties du discours reflètent l'évolution de la pensée dont elles représentent divers stades évolutifs. Pour lui, les catégories du langage participent à la formation de la pensée du locuteur et sont indissociables de celle-ci.

Notons qu'il accentue dans sa conception la «tendance analytique» qui caractérise, selon lui, la psychogenèse et qui s'accomplit par la décomposition des "perceptions globales» (celostnye vosprijatija) en des "perceptions particulières ", celles des substances et de leurs propriétés. Il illustre cette tendance avec l'exemple des «participes primitifs » (pervobytnye pričastija) (ibid. : 96) conçus par lui comme des «noms primitifs » (pervobytnoe imja) (ibid. : 94) ou encore comme « des noms-participes primitifs » (pervobytnoje imja pripričastije) (ibid. : 96). Il définit en outre ces participes comme « racines non relationnelles, qualificatives ou objectives » (ibid. : 91).

Selon Potebnia, ces «participes primitifs », dits aussi «noms-participes » ou «noms d'agent $»^{12}$, sont à l'origine de l'apparition des parties du discours ${ }^{13}$. Ces «noms-participes primitifs» (pervobytnoje imja pripričastije) fonctionnent comme des propositions simples. La «mise en syntaxe» se manifeste sur le plan psychologique par division ou par dissociation des représentations dont les «participes primitifs» étaient originellement porteurs. Chaque représentation dissociée a donné lieu à deux représentations ou à deux groupes de représentations qui ont été par la suite désignés par les termes de "sujet» et de "prédicat» (Potebnia 1958 [1874] : 96).

12 Plus tard, dans le troisième volume de ses Zapiski..., Potebnia revient sur la définition des participes primitifs, en les posant comme : «pričastije - suščestvitelnoje (participe - nom), c'est-à-dire le mot doté d'une certaine substance et d'une propriété ou d'un trait distinctif produit par cette substance, nomen agentis » ou encore comme « le nom de l'agent [imja dejstvujuščego lica] nomen agentis » (Potebnia 1968 [1899] : 82).

13 Le participe (pričastije) est conçu par Potebnia comme l'élément intermédiaire entre le nom/l'adjectif et le verbe, à mi-chemin entre les deux : dans le participe, le trait distinctif émergeant (voznikajuščij priznak), caractéristique du verbe, est représenté par ce qui est donné (predstavljaetsja dannym), ce qui constitue la caractéristique du nom. Il est pensé comme dépendant de l'énergie propre à l'objet, tandis que cette même énergie n'est pas pensée (Potebnia 1958 [1874] : 94-96). 
Les participes primitifs de Potebnia sont des éléments syncrétiques exprimant à la fois l'objet et son action. En effet, d'après l'auteur, la pensée rendue objective dans la parole et soumise au contrôle de la conscience commence non pas par la décomposition de la perception en substance et en prédicat ou attribut, mais par la désignation d'une perception globale. Ainsi, dans le verbe letit (de letet' : voler, [il] vole, quelque chose vole), le mot impliqué par la partie lexicale let- de ce verbe signifiait non pas l'action prise de façon abstraite, mais une perception encore non décomposée, une fusion indifférente de l'objet et de l'action (ou du sujet et de son action) (bezrazličnuju sovokupnost' predmeta i dejstvija). Il s'agit donc de la fusion indifférente de l'objet et de l'action, où « indifférente » signifie chez Potebnia «non analytique », «non démembrée» ou encore «non articulée ».

La conception des «participes primitifs » est la réponse de Potebnia à l'hypothèse de Steinthal, qui postule que « les premiers mots» sont « les noms des traits distinctifs » (Merkmalwörter) ou encore qu'on peut les qualifier d'adverbes, en tant que ces mots désignent des traits caractérisant des objets perçus comme en activité ${ }^{14}$. Potebnia se réfère dans ce contexte à la position de Steinthal, selon lequel « ce n'est pas l'activité elle-même qui est reflétée dans le son, mais l'impression produite par elle dans l'âme » (Potebnia 1999 [1862] : 130).

Ainsi, Potebnia présente la décomposition analytique comme un processus intrinsèque et spécifique au psychisme. Ce faisant, il se fonde sur l'idée généralement acceptée par la psychologie de son temps. Sa conception du langage, tributaire de cette thèse, s'avère par conséquent extrêmement traditionnelle. Ce même modèle explicatif du psychisme est appliqué par exemple par $\mathrm{W}$. Wundt à l'analyse psychologique de la proposition. Il semble en effet que le terme de «représentation globale» (Gesamtvorstellung) ${ }^{15}$, que Wundt utilise dans sa conception analytique de la proposition, ait pour base le même dispositif psychique que ces «participes primitifs » de Potebnia. La formation d'une unité syntaxique suit une tendance analytique du psychisme : la syntaxe est le résultat d'une décomposition de la « représentation globale » en deux éléments ou en deux représentations autonomes qui sont par la suite formalisées en termes de position : le sujet et le prédicat ${ }^{16}$.

\subsection{La syntaxe énergétique d'Ovsianiko-Koulikovski}

Le postulat essentiel de la «linguistique énergétique» de Dmitri OvsianikoKoulikovski (Dmitrij Ovsjaniko-Koulikovskij, 1853-1920), professeur à l'université de Kharkov et partisan de la doctrine de Potebnia, consiste à poser la forme grammaticale comme un moyen d'aperception ${ }^{17}$. Le résultat de cet acte aperceptif

14 Voir Steinthal $1855: 328$; Potebnia 1999 [1862] : 130.

15 Voir l'emploi du concept de Gesamtvorstellung ou de « représentation globale » par Wundt (1900, vol. 2 : 234-247).

16 On observe que les catégories grammaticales chez Potebnia se fondent sur la notion de représentation, comme c'est le cas chez Steinthal et plus tard chez Paul.

17 Ovsianiko-Koulikovski 1912 : 3-4. Cette affirmation rappelle la position de Steinthal, qui considère que c'est l'élément moyen, intermédiaire entre l'élément de départ et le nouvel élément qu'on cherche à 
est l'apparition d'une proposition grammaticale « comme une forme particulière de la pensée » (Ovsianiko-Koulikovski 1912 : 6). La définition psychologique des parties du discours énoncée par cet auteur pose également ces dernières comme «des formes particulières de la pensée ${ }^{18}$. Ces «formes mentales » servent à transformer les impressions reçues par le psychisme. Ce sont des catégories ou des rubriques toutes prêtes, des outils de classement d'impressions ou encore des outils psychologiques. Ainsi, les noms, les verbes, les adjectifs, etc., constituent pour Ovsianiko-Koulikovski diverses "formes de la pensée » (ibid. : 39-42). Selon lui, le verbe possède un statut particulier : il est la «forme de la pensée » qui représente les propriétés (priznaki) comme étant produites par l'activité des agents (sujets ou objets). Ainsi, le verbe présenterait la perspective de l'action (ibid. : 39).

Pour l'auteur, le «processus de la parole-pensée [reci-mysli]» se définit comme « un emploi syntaxique des mots : transformation des formes grammaticales en formes syntaxiques, transformation du mot en une grandeur syntaxique en général, et en partie d'une proposition en particulier » (ibid. : 6). Ce travail de « mise en prédicat» est inconscient, il s'accomplit de manière automatique, sans dépense de forces intellectuelles ni psychiques. Servant à l'« économie de forces psychiques », le travail de combinaison syntaxique est proche de l'aperception inconsciente qui caractérise le fonctionnement des formes grammaticales (ibid.). La mise en syntaxe se réalise dans la sphère inconsciente : « Les mots deviennent des grandeurs syntaxiques près du seuil de la conscience. » Selon OvsianikoKoulikovski :

L'emploi syntaxique des mots, ou ce qu'on appelle les formes syntaxiques, constitue un travail particulier de la pensée qui s'accomplit automatiquement dans la sphère inconsciente près du seuil de la conscience. Ce travail vise les formes grammaticales des mots, il est indispensable pour que les données de la conscience puissent se transformer en un acte de parole-pensée [akt reči-mysli]. (ibid. : 7)

Pris hors de cet acte de "parole-pensée », le mot ne possède pas de forme syntaxique, il n'a que la forme grammaticale qui lui est propre. La participation du mot à l'acte de parole-pensée est la propriété essentielle de la forme syntaxique et la condition indispensable pour la réalisation de celle-ci (ibid. : 8).

Ovsianiko-Koulikovski finit par définir les symptômes psychologiques qui accompagnent le phénomène de la prédication. Il appelle la prédication « un mouvement particulier de la pensée » (ibid. : 51) ou encore « une forme particulière de la pensée » (ibid.: 6,8). C'est par ce "mouvement de la pensée » que se définit la proposition : son absence signifie l'absence de toute proposition (ibid. : 51). Cette forme s'accompagne d'une « sensation intellectuelle particulière » qui est « beaucoup plus consciente [soznatel'nee] que toute autre » (ibid. : 26-27).

Cette dernière se distingue par son caractère et par le degré de clarté d'autres sensations qui accompagnent d'autres formes syntaxiques. Selon Ovsianiko-

comparer, qui joue le rôle de « moyen d'aperception » dans le processus aperceptif du psychisme (Steinthal 1858 [1851] : 93-95).

18 Ibid. : 6 et 39-49. La comparaison s'impose, là encore, avec l'idée de Paul concernant le fondement psychologique de toute catégorie grammaticale. 
Koulikovski, dans la pensée, cette "sensation intellectuelle», associée à la prédication, confère un poids particulier aux éléments prédiqués. Telle est, selon lui, la sensation du prédicat ou de la prédicativité (oščuščenie skazuemosti ili predicirovanija), ou encore, la « sensation qui accompagne l'acte d'une prédicativité grammaticale» (ǒščščenie, soputstvujuščee aktu grammatičeskogo predicirovanija) (Ovsianiko-Koulikovski 1912 : 27).

La prédication est "une forme syntaxique», mais « une forme syntaxique supérieure ou absolue ». C'est une « grandeur syntaxique qui participe à l'acte de la parole-pensée [akt reči-mysli] »; cette grandeur " résulte d'un travail intellectuel particulier qui vise à organiser ou à ordonner des mots entrant dans des combinaisons syntaxiques » (ibid. : 8), du «travail intellectuel qui établit des relations internes entre deux ou plus de deux grandeurs grammaticales » (ibid. : 6). Cette «grandeur syntaxique » est à l'origine de la «sensation intellectuelle particulière », provoquée par les poids respectifs différents de l'élément déterminé et de l'élément qui le détermine (comme par exemple, un nom et un adjectif, un nom et un verbe). Ovsianiko-Koulikovski dresse une hiérarchie des formes syntaxiques selon le type de sensations qu'elles provoquent. $\mathrm{Si}$, pour l'acte de concordance (soglasovanie), une sensation d'accompagnement n'apparait pas nécessairement, en revanche, pour l'acte de gouvernance (upravlenie), la sensation est toujours présente; enfin, la sensation qui accompagne l'acte de la prédication est la plus puissante (ibid. : 9-11).

[La] prédicativité grammaticale [grammaticeskoje predicirovanije ili skazuemost'] est le processus de la pensée qui consiste en ce que la mise en prédicat [predicirovanije] [...] est aperçue [c'est-à-dire «aperceptée »] par une forme grammaticale précise. Il en résulte la formation d'une proposition grammaticale en tant qu'une forme particulière de pensée qui, d'une part, est distincte du jugement psychologique (pré-langagier) et, d'autre part, du jugement logique (supra-langagier). (ibid. : 29)

Ovsianiko-Koulikovski, qui se veut ouvertement « disciple» de Potebnia et qui hérite à ce titre de l'apport théorique de Steinthal, reste une figure transitoire, à mi-chemin entre la méthode génétique et la méthode psychologique. Sa conception syntaxique cherche à analyser les modes selon lesquels la conscience du locuteur reflète les propriétés du monde. Sa définition génétique des parties du discours, comme le souligne Danilenko, met en évidence qu'elles sont des modes de conceptualisation du monde. Pour Ovsianiko-Koulikovski, l'instance génétique coïncide de fait avec l'instance synchronique car l'« homme moderne », quand il réalise la signification des parties du discours, reproduit le parcours mental d'autres individus qui avaient créé les premières parties du discours (Danilenko 2009 : 122). Il semble qu'il s'agisse ici d'une façon d'assimiler l'idée néo-grammairienne (et particulièrement, celle de $\mathrm{H}$. Paul) concernant la création primitive (Urschöpfung) comme création continuée dans le langage (Paul 1909 [1880] : 174-188). En effet, cette continuité, qui résulte de la continuité et de l'homogénéité du psychisme, évacue la délimitation tranchée entre la synchronie et la diachronie, en tout cas sur le plan psychique.

Rappelons que l'apport des néo-grammairiens et surtout de Paul a consisté à 
combiner la perspective génétique (diachronique) et la perspective psychologique (synchronique). C'est là qu'on voit intervenir le dispositif de l'analogie posé comme le mécanisme psychique fondamental. En effet, selon Paul, l'homme n'a pas accès aux représentations d'autrui. Pour maîtriser le sens de ses propres représentations, il doit se les construire en suivant pour cela les mêmes étapes que tout autre individu. Ainsi, Paul transpose sur le plan du langage l'idée de la psychologie « atomiste » de Herbart : ce dernier pose la non-communication entre les représentations faisant partie des consciences isolées et, par conséquent, le caractère "monadologique» des consciences individuelles. Cette construction des représentations est analogique dans la mesure où la dimension diachronique s'inscrit dans la dimension synchronique du psychisme. En d'autres termes, le dispositif analogique illustre la nature indissociablement psychophysique de l'être humain.

Pour Ovsianiko-Koulikovski, le prédicat forme le centre de la proposition car c'est lui qui est porteur de la prédicativité (predikativnost'). La proposition est une combinaison mentale particulière qui se réalise par la « mise en prédicat » ou la prédication ${ }^{19}$. Il s'agit aussi d'une tendance téléologique au sein de la syntaxe : en effet, le prédicat est posé comme le «but » de la prédication (c'est-à-dire, de la proposition). Cette vision coïncide avec la définition du rhème comme l'élément essentiel de la proposition et comme sa véritable visée communicative, définition caractéristique depuis G. Gabelentz pour le courant psychologique. Du point de vue de la « réalité » qu'on cherche à décrire, le but du prédicat chez Ovsianiko-Koulikovski consisterait à désigner le trait distinctif (priznak) produit par l'objet qu'exprime le sujet.

\subsection{La syntaxe «formaliste » de Fortounatov}

Dans la linguistique de Filipp Fortounatov (1848-1914), fondateur de l'influente École linguistique de Moscou, qui fait partie du courant de « linguistique psychologique » russe, la notion de proposition est tributaire du concept de « syntagme » (slovosočetanije). La proposition n'est qu'un cas particulier d'un syntagme, c'est un « syntagme achevé » qui s'oppose à un « syntagme inachevé », c'est-à-dire à toute autre combinaison de mot sans portée prédicative. Le "syntagme» est défini comme " une totalité qui se constitue par la combinaison d'un mot autonome sémantiquement plein avec un autre mot autonome sémantiquement plein »; formant une partie d'une proposition, cette combinaison a lieu d'abord dans la pensée et ensuite seulement dans la parole ( $r e c ̧$ ). Il s'agit d'une entité essentiellement psychique : en effet, elle consiste avant tout en une combinaison de « représentations », car le « syntagme » ne fait que manifester l'état mental du locuteur.

Selon Fortounatov, ce «syntagme » employé dans la parole exprime une combinaison d'une représentation d'un mot avec une représentation d'un autre mot réalisée dans la pensée du locuteur. Pour l'interlocuteur, cette combinaison 
des mots dans la parole d'une autre personne est un signe d'une combinaison des représentations qui s'est produite dans la pensée de celui qui parle (Fortounatov 1956, vol. $1: 131$ et 162). Ainsi, aussi bien pour le locuteur que pour l'interlocuteur, le «syntagme" est opérationnel par rapport aux « représentations des mots ». Le "syntagme achevé », opposé à un «syntagme inachevé », signifie pour Fortounatov une proposition à deux termes principaux (polnoje predloženije). Dans le "syntagme achevé », un mot se combine avec un autre mot « comme une partie d'un jugement avec une autre partie du jugement» (ibid. : 162-163). Le jugement étant posé comme critère des "syntagmes achevés », l'«achèvement» du syntagme est tributaire de la pensée où ce processus s'accomplit.

Fortounatov souligne que l'analyse grammaticale d'une proposition ne coïncide pas nécessairement avec son analyse psychologique. En effet, l'analyse du premier type s'attache aux significations des mots dans le cadre des syntagmes et rend compte de la formation de ces derniers. Tout syntagme déjà formé en tant que proposition peut être reproduit par la suite dans la pensée comme une partie d'une nouvelle proposition formée dans la pensée. Du fait que le contenu d'un syntagme peut être la reprise d'un élément déjà connu dans le discours et ainsi figurer comme sujet psychologique, l'analyse grammaticale n'épuise pas l'analyse psychologique. En effet, cette dernière ne permet pas d'établir si le sujet grammatical ou le prédicat grammatical correspondent réellement au sujet psychologique ou au prédicat psychologique dans la pensée du locuteur (Fortounatov 1956, vol. $2: 456$ ).

L'auteur accentue ainsi le clivage entre ce qu'il appelle une «proposition grammaticale» (gramamtičeskoje predloženije) et un «jugement psychologique » (psixologičeskoje suždenije), entre une formation grammaticale et un emploi dans la parole ( $v$ reči $)$. Il propose l'exemple suivant : le syntagme (slovosočetanije) « N. N. est venu de Moscou » (N. N. priexal iz Moskvy), examiné du point de vue de sa formation, est une « proposition grammaticale » où le syntagme «de Moscou» est un complément circonstanciel qui complète le prédicat grammatical «est venu». En revanche, dans le «jugement psychologique» exprimé par cette proposition dans la parole, les représentations des constituants du syntagme «de Moscou» auraient pu être le prédicat. Dans ce cas, les représentations liées au syntagme «N. N. est venu» auraient pu former le sujet $\mathrm{du}$ « jugement psychologique » si le fait de la venue de N. N. de quelque part était préalablement connu. Ainsi, diverses parties dépendantes d'une proposition, conçues comme compléments au niveau de leur formation grammaticale, peuvent être reproduites dans la pensée comme des parties principales ou dominantes. Cette possibilité est liée aux remaniements que les relations entre les représentations des mots peuvent subir dans la pensée (ibid. : 456-457).

Pour Fortounatov, dans les « jugements psychologiques » ou dans les « propositions dans la pensée », une partie au moins comporte une représentation du mot (ou des mots). C'est la raison pour laquelle il convient de distinguer dans les «propositions exprimées dans la parole» les propositions complètes et les propositions incomplètes. Ce dernier cas est l'expression d'un «jugement 
psychologique » où seulement une partie sur deux comporte une représentation du mot (ou des mots). La seconde partie, en revanche, ne possède pas une représentation verbale, elle consiste en une représentation immédiate (neposredstvennoje predstavlenje) de l'objet de la pensée ou de sa partie. Une « proposition incomplète " se trouve exprimée dans la parole dans les cas où le locuteur combine à l'intérieur d'une proposition une sensation en provenance de son expérience antérieure (accompagnée d'une représentation correspondante) avec une représentation engendrée par l'expérience donnée. Ces deux représentations se combinent par association, laquelle est tributaire des expériences antérieures (ibid. : 448-449).

Fortounatov donne comme exemple le mot « feu » qui peut s'employer comme une proposition incomplète. Dans le «jugement psychologique » que cette proposition exprime dans la parole, la représentation du feu, de la fumée que le locuteur vient de percevoir, constitue le «sujet psychologique». Quant à la représentation du mot « feu », elle fait partie de la seconde partie de la pensée et constitue le "prédicat psychologique ». De la même manière, d'autres mots peuvent exprimer dans la parole les "prédicats psychologiques» dans les «jugements psychologiques» (ou les «propositions dans la pensée ») qui ont pour « sujet psychologique » une « représentation immédiate » de certains objets. Par exemple, chacun des mots tels que «maison» $($ dom $)$, «arbre» (derevo), « oiseau » (ptitsa) peut former dans la parole une proposition incomplète de ce type (ibid. : 450-451).

On reconnaît facilement dans la conceptualisation des «propositions incomplètes » par Fortounatov les développements de H. Paul relatifs aux «interjections primordiales » et à leur rôle dans la « création primitive » du langage (Paul 1909 [1880] : 183-184).

Dans le chapitre des Prinzipien consacré à la « création primitive » (Urschöpfung), Paul s'attarde sur le dispositif psychologique à la base de l'émergence du langage. Il accepte la possibilité de l'existence d'un groupe d'interjections qui a surgi, selon toute évidence, à cause des mouvements réflexes. La plupart de ces interjections sont des réactions aux excitations momentanées des nerfs auditifs et visuels. D'origine réflexe, ces interjections se trouvent réemployées dans les souvenirs et dans les récits des événements qui se manifestent sur le plan psychophysiologique par de telles excitations momentanées. Dans le cas des formations langagières "primordiales", on ne peut parler de formations par analogie. La langue primitive est dépouillée de catégories grammaticales, ses phrases primitives correspondent à des visions globales. L'exemple en est donné par les phrases nominales : voleur! feu! Elles sont, comme les mots en question, des prédicats dont le sujet est une impression physique. Pour que l'homme puisse énoncer de telles phrases, il faut qu'il isole un élément particulier de la diversité des impressions qui remplissent simultanément ses sens. Mais comme cette séparation d'un élément ne peut pas encore être réalisée par une opération logique, il faut qu'elle soit provoquée par le monde extérieur. Il faut que se produise quelque chose qui fixe l'attention dans une direction particulière. Ce n'est pas un monde silencieux et calme, mais un monde animé et bruyant dont l'homme a pris 
conscience. Ces mots primitifs ont lié une impression inachevée à une interjection (ibid.).

Ainsi, Fortounatov «syntaxise » le dispositif psychique décrit par H. Paul et fonde clairement une unité de sa typologie syntaxique - les propositions incomplètes - sur le critère psychologique qui en fait des " prédicats psychologiques ", des « impressions physiques » ou des "représentations immédiates » préexistantes conçues sous forme de « sujets psychologiques ».

\subsection{Pechkovski : la syntaxe volontariste}

Aleksandr Pechkovski (Peškovskij) (1878-1933), professeur moscovite qui considère $\mathrm{F}$. Fortounatov comme son maître à penser, va encore plus loin dans sa psychologisation de la syntaxe. En effet, sa conception syntaxique est autant une conception psychologique. Cette psychologisation radicale s'accomplit à l'aide du concept de prédicativité. Pechkovski appelle "prédicativité» (skazuemost') « cette nuance dans le mot qui montre que ce mot correspond non seulement à une représentation, mais à une pensée entière » (Pechkovski 2001 [1938] : 172). Pour lui, « la prédicativité est la plus importante des catégories grammaticales, car on y trouve un enchaînement étroit de la parole et de la pensée $»^{20}$.

La syntaxe de Pechkovski exprime très clairement une position volontariste. Il écrit en effet :

La psychologie nous apprend que le processus de la pensée se distingue d'une simple association des représentations par le fait que, dans la pensée, c'est nous qui combinons nos représentations, et ce ne sont pas les représentations qui se combinent en nous. On trouve [dans la pensée] un choix conscient des représentations et un contrôle conscient de la correspondance de ces représentations avec leurs relations à la réalité [...], un travail a lieu ici (ce n'est pas par hasard que certains sont trop paresseux pour penser!), et tout travail est lié à une impulsion volontaire. (ibid. : 175)

Selon l'auteur, « la pensée est une combinaison active des représentations »: le verbe en tant que "forme mentale » transmettrait « une attitude active» du psychisme vis-à-vis des objets qui le complètent (ibid. : 176).

C'est pour cette même raison que le verbe exprime pleinement la pensée car « le verbe exprime l'action, c'est-à-dire une attitude active de l'objet à l'égard de son trait distinctif. C'est sans doute précisément cette relation des représentations [...] qui aurait dû fournir le premier matériau de la pensée, créer la pensée langagière [jazykovaja mysl'] » (ibid.). Cette «attitude active» relève de l'effet de la volonté dans la proposition ${ }^{21}$. Cet effet est tributaire de l'«élément volontaire » (volevoj ottenok) dont le verbe est le porteur privilégié et qui, selon Pechkovski, fait partie de la signification verbale. Le verbe, par sa nature même, serait une combinaison d'une représentation (faisant partie de son sens lexical) avec cette nuance volontaire (ibid. : 175) ou intentionnelle ${ }^{22}$. 
Ainsi, le modèle de la syntaxe psychologique élaboré par Pechkovski propose une relation entre le prédicat et la volonté ; il s'insère par là dans les débats d'alors portant sur la nature de l'aperception et plus largement des mécanismes de la « conscience verbale».

\subsection{A. Chakhmatov : du jugement psychologique à la « communication »}

Dans le premier paragraphe de sa Syntaxe du russe, Alekseï Chakhmatov [Alexej Šakhmatov] (1864-1920), membre de l'Académie impériale de St Pétersbourg, lui aussi partisan de l'orientation psychologique en linguistique, appelle syntaxe « la partie de la grammaire qui étudie les modes de manifestation de la pensée dans le langage [ $v$ slove $]$, c'est-à-dire dans l'ensemble des signes externes reproduits par les organes de la parole et perçus par l'ouïe » (Chakhmatov 2007 [1925] : 17).

Pour Chakhmatov, génétiquement, le « langage avait pour but la communication » (kommunikacija). Il entend par la « communication» toute « combinaison des représentations » que le locuteur cherche à transmettre dans un acte de parole. En effet, pour lui, la visée de la communication consiste toujours à transmettre non pas « des noms de représentations isolés mais des combinaisons de représentations » (ibid.).

Ces « combinaisons de représentations » correspondent aux "états d'âme » (duševnye pereživanija) du locuteur et révèlent ses actes de pensée. Par conséquent, selon Chakhmatov, « dès le début, le signe du langage a reçu non pas des perceptions, sensations, sentiments de la volonté [du locuteur]» mais il a assimilé « des combinaisons des représentations qui leur correspondent». Ces combinaisons de représentations, exprimées dans les signes du langage, ont surgi «dans l'âme du locuteur» comme "autant de manifestations de sa volonté », pour exprimer une affirmation ou une négation de quelque chose. C'est seulement plus tard, par décomposition de ces signes langagiers des combinaisons simples ou complexes, qu'on a vu se former des mots qui désignent des représentations isolées.

Chakhmatov appelle la "parole» (reč) « une expression verbale des combinaisons de représentations »; il conçoit la « parole » comme une manifestation des «enchaînements [sceplenija] de représentations, qui se succèdent continuellement dans l'âme du locuteur». Il appelle par ailleurs "propositions » (predloženija) «les parties de la parole humaine qui correspondent à des combinaisons particulières des représentations ». Selon lui, dans le langage, on a vu d'abord apparaître des propositions ; ensuite, « par la voie de décomposition, fondée sur leur comparaison et leur influence réciproques, on a vu s'extraire, à partir d'elles, des syntagmes et des mots aptes à s'employer sur un mode autonome » (ibid. : 19). Cet usage reste très réduit et accidentel, car habituellement, les mots et les syntagmes fonctionnent dans le cadre des propositions.

Chaque verbe comporte cette «nuance d'une activité consciente» (ottenok soznatelnoj dejatelnosti), chaque verbe exprime une tendance ou une visée, c'est un élément qui " anime » tout autre élément auquel il s'applique. Ce statut « vivant» des verbes remonte à l'époque de l'animisme primitif qui a également conditionné l'émergence de la « nuance objective » (ottenok predmetnosti), caractéristique des noms ( $\mathrm{ibid}$. : 99-100). 
La tâche de la syntaxe consiste à étudier à la fois (1) les propositions comme des manifestations verbales des unités de la pensée, et (2) les syntagmes et les mots dans la mesure où ces derniers n'ont pas perdu leurs liens avec les premiers, en gardant la signification de leurs parties ou de leurs membres. Restent hors de la syntaxe les éléments qui ont perdu tout lien avec les propositions et qui sont devenus indépendants des éléments de leur entourage par leurs formes et leurs significations (Chakhmatov 2007 [1925] : 18).

Après avoir défini la combinaison qui constitue la nature de la proposition, Chakhmatov passe à la " communication » comme «fondement psychologique de la proposition » (ibid. : 19). À partir de l'idée d'origine néo-grammairienne du « fondement psychologique des parties du discours $»^{23}$, il pose la « communication » comme la base psychologique de la proposition. La définition psychologique de la proposition est celle d'" une unité de la parole perçue par le locuteur et par l'interlocuteur comme une totalité grammaticale [grammatičeskoje celoje] qui sert à exprimer verbalement une unité de la pensée » (Chakhmatov 2007 [1925] : 19).

Selon l'auteur, « le fondement psychologique de notre pensée est la réserve des représentations, fournie par toute l'expérience antérieure, qui s'élargit en permanence par nos vécus en train de s'accomplir ». Ainsi, il retient la définition du psychisme issue de la psychologie herbartienne et selon laquelle, rappelons-le, l'existence même de nouvelles représentations est tributaire de l'interaction des perceptions passées et présentes (voir notre note 10).

Pour Chakhmatov, la base psychologique de la proposition consiste à combiner ces représentations dans un acte particulier de la pensée visant à communiquer aux autres les combinaisons ainsi construites (ibid.).

\section{La notion de « communication »}

Le mécanisme de la « communication » (kommunikacija), d'après Chakhmatov, consiste à transférer une saisie momentanée des représentations dans la pensée du locuteur vers la conscience de l'interlocuteur. La volonté joue un rôle très important dans la construction prédicative: c'est elle qui combine deux représentations, en établissant entre elles un lien prédicatif. Par conséquent, la prédication est le produit de la volonté qui conditionne cette saisie ou cette combinaison; ses effets se retrouvent derrière toute mise en forme prédicative (Chakhmatov 2007 [1925] : 19).

Selon l'auteur, la « communication élémentaire » (qui est aussi « l'unité de la pensée la plus simple ») consiste en la combinaison de deux représentations. C'est le mouvement de la volonté qui établit un lien prédicatif entre les deux représentations ${ }^{24}$. Chakhmatov définit comme «communication» toute combinaison des représentations qui s'accomplit de façon intentionnelle ou intellectuelle (ibid.). En d'autres termes, est «communication» toute combinaison

23 Sans doute un renvoi à Paul (1909 [1880] : 263).

24 Ibid. Rappelons que Ovsianiko-Koulikovski définit le «lien prédicatif» comme « causal » et « génétique » (Ovsianiko-Koulikovski, Syntaxe du russe, dans Ščeulin \& Medvedeva 1965 : 331). 
réalisée avec une intention pragmatique ${ }^{25}$. Il précise dans le paragraphe 3 que la « communication»s'exprime dans des propositions. La «parole interne » est l'intermédiaire habituel entre la communication et la proposition verbale. Il définit la « parole intérieure » comme la pensée manifestée à l'aide des signes auditifs et, partiellement, des signes visuels. Selon lui, c'est dans la parole intérieure qu'on voit se concrétiser ces images complexes et instables qui se combinent dans la «communication». La parole intérieure emprunte son matériau essentiel aux représentations phoniques des mots : par conséquent, la parole intérieure utilise les signes auditifs ou phoniques (ibid. : 20).

Mais la «communication », qui est initialement le travail de la pensée, peut confier à la parole interne d'autres éléments que les signes auditifs : ce sont des représentations des relations et des sentiments provoqués par la combinaison des représentations dans la communication (ibid.). Par l'intermédiaire de la parole interne, ces autres éléments tendent à se manifester dans la parole externe (ibid.). Ces développements semblent renvoyer (sans s'y référer explicitement) aux discussions d'alors autour du «sentiment de la forme», caractéristiques du contexte germanophone.

Ainsi, on voit se produire dans la pensée et dans la parole interne une décomposition des images complexes. C'est uniquement grâce à la décomposition des images combinées que la communication devient déterminée et parvient à se manifester ${ }^{26}$. Ainsi, pour Chakhmatov, l'acte de communication résulte d'un processus psychique complexe. Il débute par le mouvement de la volonté qui vise à transmettre au locuteur cette combinaison de deux représentations. Il continue ensuite par l'analyse psychique de ces représentations (ibid.).

Dans le paragraphe 4 , Chakhmatov réserve aux deux parties du jugement psychologique les «termes latins » de "sujet» et de "prédicat», tandis que les termes équivalents russes podležašee et skazuemoe sont réservés aux catégories grammaticales : au sujet et au prédicat grammaticaux. Ces derniers se trouvent non seulement dans le jugement psychologique, mais aussi dans d'autres « communications psychologiques »: l'une des deux représentations combinées sera « sujet» et la seconde « prédicat» (ibid. : 21).

Chakhmatov définit le « sujet psychologique » comme la représentation qui domine l'autre représentation combinée avec elle, cette seconde représentation dominée étant définie comme le "prédicat psychologique ». Ainsi, le terme de « représentation dominante» (Wundt dit dominierende Vorstellung) montre que la seconde représentation se trouve en liaison causale ou en liaison de dépendance avec la première représentation.

Il s'agit d'une " domination objective », conditionnée par la nature même de la première représentation, et d'une « dépendance objective », conditionnée par la nature de la seconde représentation (ibid. : 21-22). Chakhmatov note le cas où a lieu la coïncidence du sujet et du prédicat grammaticaux (podležaščee et

25 Il semble que pour Jakobson cette large notion coïnciderait avec la fonction conative.

26 On reconnaît dans cette description de la communication le mécanisme équivalent au dispositif de la décomposition des représentations que Wundt pose au fondement du psychisme. 
skazuemoe) avec le sujet et le prédicat psychologiques (Chakhmatov 2007 [1925] : 21-22). Néanmoins, selon lui, c'est avant tout la nature des représentations qui détermine leur statut et qui fonde leur caractère déterminant ou déterminé : entre ces deux groupes des représentations, il existe des relations déterminées par rapport à la possibilité d'exprimer le « sujet» ou le «prédicat».

Pour Chakhmatov, il en découle le principe de combinaison suivant :

Lors de la combinaison d'une représentation de l'objet avec une représentation de la propriété ou du trait distinctif, la première représentation, celle de l'objet, parce qu'elle est dominante par nature à l'égard de la propriété, sera toujours le sujet, tandis que la seconde représentation sera toujours le prédicat. (ibid. : 22)

Ainsi, dans sa définition des parties du discours, il se réfère aux propriétés intrinsèques des langues : les noms des objets au nominatif formeront nécessairement le sujet grammatical par rapport à des verbes ou à des adjectifs combinés avec ces premiers (ibid.). Ce principe relève des propriétés des représentations auxquelles correspondent les formes grammaticales : c'est ainsi qu'on voit se manifester le lien interne entre la langue et la pensée, entre les catégories grammaticales et les catégories psychologiques (ibid. : 22-23).

\section{Le prédicat et le sujet psychologiques}

Chakhmatov considère par la suite les cas de non-coïncidence entre « les formes langagières des propositions et la communication psychologique ». Pour lui, cette non-coïncidence n'implique pas une contradiction : « les formes grammaticales », explique-t-il, « ne peuvent pas entrer en contradiction avec la nature psychologique des représentations qui leur correspondent» (ibid. : 23). Nous allons voir pourquoi.

Chakhmatov rejette l'idée du prédicat et du sujet psychologiques telle qu'elle est formulée par G. von der Gabelentz et par H. Paul. Selon lui, dans toutes les conditions, dans les phrases du type « l'oiseau vole » (ptitsa letit) et « la mère est malade » (mat' bol'na), ce sont toujours « l'oiseau » et « la mère » qui seront le sujet, indépendamment de tout accent mis sur tel ou tel élément. De même, dans les jugements psychologiques correspondants, le sujet ou la représentation dominante sera toujours la représentation de «l'oiseau » ou de « la mère », tandis que les propriétés ou les traits distinctifs comme « vole », " est malade », formeront toujours la représentation dominée (ibid.).

Dans le paragraphe 6, Chakhmatov se démarque explicitement des positions de Gabelentz, de Paul et de Fortounatov. Après les avoir résumées, il mentionne la « position particulière » de Wundt qui n'a pas suivi cette «nouvelle définition » des parties du jugement psychologique. En effet, Wundt n'a pas accepté la transposition des termes logiques sujet - prédicat en psychologie. Chakhmatov semble adhérer globalement à la position wundtienne. Ainsi, il reprend l'exemple analysé par Wundt - « aujourd'hui, c'est mon anniversaire »- pour citer l'avis du psychologue allemand selon lequel, en dépit de tout accent possible placé sur l'élément « aujourd'hui », le fondement aussi bien logique que psychologique de ce message réside dans la représentation « anniversaire ». Wundt a retenu le terme 
de « représentation dominante » (dominierende Vorstellung) pour la représentation qui correspond à l'élément sur lequel se concentre l'attention (ibid. : 23-24).

Pour Chakhmatov, la question principale porte sur la possibilité même d'utiliser les termes logiques « sujet» et « prédicat» en psychologie (ibid. : 24). Selon lui, il serait possible d'employer ces termes en psychologie au sens plus large, en les étendant à toutes les relations psychologiques, sans se limiter au jugement psychologique. Dans ce contexte, il définit le "sujet psychologique » comme la représentation qui, par sa nature, domine la représentation en position de prédicat (ibid. : 24-25).

Dans ses développements ultérieurs, on voit revenir la notion de volonté qui, décidément, constitue une référence importante de la syntaxe psychologique en gestation. Selon lui, la définition du «sujet psychologique » dans l'esprit de Gabelentz suggère deux types de représentations dominantes : celles qui le sont « par nature » et celles qui le sont « par la volonté du locuteur».

Pour Chakhmatov, la position de Gabelentz et de ses partisans conduit à l'idée selon laquelle il existerait dans la pensée des représentations qui, sans être dominantes par leur nature, le deviennent soit en fonction de l'humeur du locuteur, soit en fonction de telle ou telle orientation qu'il confie à son message. Ainsi, on pourrait postuler l'existence dans la pensée, à côté des représentations dominantes par leur nature, de représentations qui sont dominantes selon la volonté ou l'humeur du locuteur. Chakhmatov propose alors de réserver le terme de «sujet psychologique » aux représentations de premier type, celles qui sont dominantes «par leur nature », et le terme de «représentation dominante » aux représentations de second type (ibid. : 25).

L'auteur perçoit l'émergence de ces deux types de représentations comme un conflit naturel entre les fondements du psychisme humain et ses états instables ou passagers. Mais, pour lui, ce conflit est très limité : le plus souvent, il se résout au profit des relations fondamentales entre divers groupes de représentations plutôt qu'au profit de la volonté du locuteur (ibid.).

Dans le paragraphe 7, Chakhmatov précise que le nom de l'objet dans une proposition sera toujours un sujet grammatical par rapport à un verbe ou à un adjectif qui se combinent avec lui. De même, dans la pensée, la représentation de l'objet sera toujours le sujet psychologique par rapport à la représentation d'une propriété (ou d'un trait distinctif), ce dernier élément étant pensé comme un prédicat psychologique (ibid.).

En se référant au célèbre postulat des Prinzipien de Paul, qui posent la base psychologique des catégories grammaticales, Chakhmatov défend l'idée d'une « coïncidence suffisante » des catégories grammaticales et psychologiques dans le langage (ibid. : 25-26).

Ainsi, on trouve chez Chakhmatov la même hésitation qui caractérise l'évolution de la pensée de Steinthal : l'hésitation entre le modèle logique du jugement et le modèle psychologique ${ }^{27}$. Mais en même temps, l'attitude de Chakhmatov

27 En particulier, Steinthal montre une hésitation analogue dans la question des propositions nominales ou impersonnelles. Ce dualisme de la position de Steinthal s'exprime clairement dans sa discussion avec F. Miklosich (Graffi $2001:$ 60-62). 
permet de mieux comprendre l'émergence de la composante pragmatique à partir des discussions propres au courant psychologique. En effet, certaines prémisses au sein des tendances psychologiques conditionnent ce passage à la «communication » qui caractérise à la fois le champ germanique et le champ russe. Les positions de Steinthal et de Chakhmatov convergent dans l'idée selon laquelle toute proposition exprime une communication, cette dernière étant toujours à deux éléments (Chakhmatov 2007 [1925] : 28). Ainsi le dualisme logique du «thème propos » cède la place au dualisme pragmatique « thème - rhème ».

\section{WUNDT ET LA « SYNTAXE VOLONTARISTE » DANS LE CHAMP RUSSE}

Les conceptions syntaxiques formulées au sein du courant psychologique russe (et, particulièrement, par Ovsianiko-Koulikovski, Pechkovski et Chakhmatov) associent la combinaison d'éléments langagiers avec l'effet psychique particulier définissable comme volonté : elles sont de nature volontariste.

Cette persistance de l'instance volontaire dans les définitions de l'objet syntaxique incite à supposer une référence psychologique. À cet égard, ces conceptions syntaxiques semblent parfaitement tributaires du concept de l'« aperception volontaire » de Wundt (1832-1920). En effet, la notion de volonté est fondamentale dans la psychologie et la philosophie de ce dernier. C'est la définition « volontariste » ou « active » de l'aperception par Wundt qui détermine la différence entre son modèle du psychisme et celui de Herbart. Chez Wundt, la volonté est décrite comme le fondement du psychisme, elle est le principe explicatif des actions intentionnelles mais aussi de l'évolution de la vie organique (Wundt 2002 [1902-1914] : 559-568).

Selon l'auteur, le corps vivant se développe comme le résultat d'une somme d'actes intentionnels isolés. L'apparition d'un organisme est un processus volontaire : c'est toujours une auto-création qui n'est guidée par aucune idée subjective d'un but préalable. Pour Wundt, la volonté possède une valeur transformatrice. Les « forces vitales » se libèrent dans les êtres vivants, ces forces émancipées gouvernent l'évolution des organismes vivants eux-mêmes. En ce sens, on peut considérer l'ensemble des êtres organiques comme l'émancipation de la volonté dans la nature. Un acte volontaire se trouve à l'origine de toute créature organique. Toute réaction volontaire à l'égard des excitations externes porte en elle les germes d'un développement ultérieur des organes qui y participent. La volonté en tant que principe explicatif des actions intentionnelles s'oppose à l'idée d'une force aveugle qui gouvernerait l'évolution. À la différence de celle-ci, la nouvelle forme d'organisation, la forme organique, surgit comme « forme volontaire ». L'organisme en tant que «nouvelle forme d'organisation » de l'ensemble psychique et corporel apparaît comme un système volontaire, comme un produit de sa propre volonté initiale (voir ibid. : 666-703 et 734-764).

L'influence de la psychologie volontariste de Wundt en Russie s'est constituée par des voies classiques, à commencer par des séjours de formation et de recherche dans les universités et les laboratoires allemands. La destination privilégiée des jeunes psychologues russes a naturellement été le laboratoire de 
Wundt à Leipzig (fondé en 1879). Un autre élément marquant de ce transfert est l'importance des traductions en russe des ouvrages psychologiques allemands. Ainsi, pas moins de vingt-trois ouvrages de Wundt ont été publiés en Russie entre 1868 et 1922, chiffre considérable si l'on songe que trois d'entre eux ont été, en tout et pour tout, traduits en français au cours de la même période. À partir de 1910, on voit paraître en russe ses ouvrages portant sur les fondements psychologiques de l'art et du langage ${ }^{28}$.

Ainsi, les définitions de la syntaxe proposées par les linguistes russes appartenant au courant psychologique (Ovsianiko-Koulikovski, Pechkovski, Chakhmatov) expriment très clairement une vision volontariste. Cette définition $\mathrm{du}$ fonctionnement de la pensée évoque nécessairement la définition de l'aperception chez Wundt. En effet, c'est précisément la participation de la volonté à la représentation qui distingue la conception wundtienne de l'aperception de celle de Herbart. Chez ce dernier, la construction des associations est pensée sans participation de la volonté ni de la conscience, comme un processus hautement «automatisé ». En revanche, Wundt formule une conception active de l'aperception qu'il qualifie de " volonté aperceptive».

Dans le contexte allemand, l'aspect volontariste de la syntaxe psychologique n'est pas accentué. Cela s'explique par le fait que les conceptions allemandes dans ce domaine se fondent sur le modèle aperceptif herbartien, modèle «passif » ou « inconscient », où la dominante volontariste est absente.

En revanche, dans le contexte russe, la dominante volontariste se trouve fortement accentuée. À notre avis, ce fait est lié à l'influence du modèle aperceptif de Wundt, celui de l'aperception « active » ou « volontaire».

Ainsi, les conceptions russes dans ce domaine se construisent au croisement de deux lignes :

(1) la ligne du herbartianisme classique qui vient des linguistes-psychologues allemands (Steinthal, Gabelentz, Paul);

(2) la ligne de l'aperception volontaire de Wundt, dont l'influence reflète la place importante que sa psychologie et sa philosophie occupent en Russie d'alors.

Dans le contexte russe, on peut constater une tendance consistant à faire du prédicat non seulement la propriété psychologique essentielle de la proposition mais aussi une propriété de la pensée. Il en découle une substantialisation de la syntaxe, réalisée par l'introduction de la notion originellement grammaticale de prédicat dans la conscience à titre de son élément constitutif. Les théoriciens russes de la syntaxe psychologique semblent chercher à «naturaliser » cette notion de grammaire, en la transformant en un «élément $»^{29}$ de la conscience et,

28 En voici quelques exemples : Sistema filosofii [Système de la philosophie], 1902, Saint-Pétersbourg ; Osnovy iskustva [Fondements de l'art], 1910, Saint-Pétersbourg; Jazyk. Narodnopsikhologičeskaja grammatika [Langage. La grammaire de la psychologie des peuples], 1910, Kiev ; Problemy psikhologii narodov [Problèmes de la psychologie des peuples], 1912, Moscou ; Fantazija kak osnova iskusstva [L'imagination comme fondement de l'art], 1912, Moscou. Sur l'influence de Wundt en Russie, voir Romand \& Tchougounnikov 2009.

29 Le terme d' « élément » est compris ici au sens de la psychologie physiologique de l'époque, comme une unité qualitative indécomposable constitutive de la conscience. 
par conséquent, du psychisme. Le prédicat devient alors une «prédicavité » ou skazuemost', autrement dit, il est conçu comme une manifestation d'une tendance psychique générale. Il se révèle alors l'incarnation et l'expression de la stabilisation de l'énergie syntaxique dont il traduit la présence et l'effet.

Ainsi, on constate dans les conceptions russes de la syntaxe psychologique une démarche qui vise à hypostasier le dynamisme phrastique. Il s'agit de transformer le verbe en "verbalité » et le prédicat en "prédicativité ». Le concept de prédicativité (skazuemost') de Pechkovski semble dérivé du concept de verbalité (glagolnost') chez Potebnia et de prédicativité (predikativnost') chez OvsianikoKoulikovski.

Ce couple notionnel corrélatif - «verbalité » (glagolnost') et la notion de « prédicativité » (skazuemost') - comporte plusieurs composantes qui témoignent de diverses étapes de l'évolution des idées syntaxiques en Russie. Il résume ainsi la modification de l'idée de syntaxe durant la seconde moitié du XIX ${ }^{\mathrm{e}}$ siècle. Parmi ces composantes, on trouve les éléments suivants :

- le modèle génétique de Potebnia, pour qui la syntaxe reflète les étapes de la croissance morphologique du langage ;

- le modèle énergétique d'Ovsianiko-Koulikovski, pour qui la syntaxe reflète une tendance essentielle du psychisme consistant à dynamiser les représentations et à faciliter l'exercice de la pensée à l'aide du dispositif aperceptif (manifesté par les formes grammaticales);

- le modèle volontariste de Pechkovski, pour qui toute mise en syntaxe, toute combinaison de représentations est conditionnée par un effort volontaire.

\section{L'APPORT RUSSE À L'ORIENTATION PSYCHOLOGIQUE EN LINGUISTIQUE}

La théorisation de la syntaxe psychologique en Russie a marqué dans le pays l'étape décisive de la psychologisation des sciences du langage. Ce processus a suivi la tendance élaborée au sein de la linguistique psychologique allemande : au niveau de la méthodologie, elle se manifeste comme un transfert graduel du plan morphosyntaxique sur le plan psychologique. Cette dissolution de la morphosyntaxe dans le matériau psychique, inaugurée par Humboldt et imposée par Steinthal, a eu des conséquences considérables sur la tradition linguistique russe. Les traces de cette démarche restent opérantes jusqu'à nos jours.

La comparaison des idées successives dans le domaine de la syntaxe psychologique montre des modalités concrètes de la psychologisation des sciences du langage en Russie. Telle est, par exemple, la tendance «caractéristique » des langues indo-européennes, décrite par Potebnia en termes de transition allant de l'étape nominale (celle dominée par le nom) vers l'étape verbale (celle dominée par le verbe).

Il s'agit de l'abandon successif et graduel des représentations, que la tradition psychologique relie étroitement aux noms, au profit des relations, que cette même tradition associe essentiellement aux verbes ou aux prédicats. Cette émergence de 
la période prédicative correspond pour Potebnia à la croissance de l'unité de la proposition. Elle reflète également la même tendance analytique propre au psychisme qui se manifesterait dans le passage de la vision synthétique à la vision analytique, tendance étendue par l'approche évolutionniste à l'ensemble des sciences humaines. Cette virtualisation de l'objet langagier ainsi que l'évacuation des éléments substantiels ont contribué à l'essor de la syntaxe psychologique. En effet, cette dernière définit le rhème ou le prédicat psychologique comme un élément dynamique et essentiel dans toute communication. En outre, l'importance de l'élément rhématique reflète la loi fondamentale du psychisme (à cet égard, il suffit de se rappeler le statut des prédicats psychologiques chez Steinthal, Gabelentz, Paul et Vygotski).

Sur le plan de la méthode, cette tendance définie comme passage du nom au verbe semble suivre la réflexion linguistique de cette période, réflexion qui consiste à déplacer l'objet d'étude ainsi que le centre d'analyse de la diachronie vers la synchronie ${ }^{30}$. La vision "énergétique» du langage contribue à sa « désubstantialisation » : cette position est caractéristique de la linguistique psychologique qui poursuit la révision systématique de la conception morphologique héritée du comparatisme.

Ainsi, l'énergétisme d'Ovsianiko-Koulikovski se manifeste en particulier dans l'accent mis sur le verbe (le prédicat), sur la tendance à la « verbalité » (glagolnost') ou au caractère verbal. Selon Potebnia, ce « caractère verbal » (« verbalité » ou encore glagolnost') est caractéristique des langues indo-européennes ${ }^{31}$. L'énergétisme d'Ovsianiko-Koulikovski accentue l'instance dynamique de la syntaxe : pour lui, le pôle verbal ou le prédicat marquent la tendance à la libération de l'énergie propre du psychisme - ou encore, dans son optique psychologisante, on assiste à la libération de l'énergie spécifique propre au langage. En s'inspirant du principe de la transformation et de la conservation de l'énergie, en suivant la piste des sciences de la nature, celle de la transformation de la matière, le raisonnement énergétique en linguistique a pour but de libérer la forme du langage de la substance langagière. Telle est la portée de la notion de « forme syntaxique » d'Ovsianiko-Koulikovski, conçue comme le résultat du transfert de la forme morphologique ou grammaticale du mot dans une position syntaxique au sein d'une proposition ${ }^{32}$. Pour l'auteur, les formes syntaxiques se situent du côté des

30 En outre, cette transformation prédicative du nom semble renvoyer implicitement à l'idée de Steinthal affirmant le statut originellement prédicatif du nom primitif. En effet, pour Steinthal, tous les mots originaires sont des prédicats (Bumann 1965 : 85). Il est également instructif de comparer cette idée avec l'affirmation plus tardive de Paul sur le statut nécessairement prédicatif des interjections primitives, définies par lui par rapport au "sujet psychologique » qu'est la situation même où ces interjections sont émises (Paul 1909 [1880] : 182-183). L'idée de Vygotski sur la composition de la parole interne au moyen de « prédicat purs » semble découler de ces débats antérieurs ; ses origines se trouvent très probablement dans les intenses lectures de Potebnia faites par Vygotski lors de l'élaboration de sa propre conception de l'évolution psychique de la conscience langagière (Vygotski 1996 [1934] : 333-335).

31 Danilenko parle dans ce contexte de «l'accent mis sur le prédicat» ou du «predicatocentrizm » des conceptions syntaxiques de ce type (Danilenko 2009 : 110-112). Le «predicatocentrizm » est ici l'aboutissement de l'idée du prédicat comme la partie la plus productive du langage (ibid. : 112).

32 Voir Ovsianiko-Koulikovski, Syntaxe du russe, dans Ščeulin \& Medvedeva 1965 : 317. 
automatismes et de l'inconscient. C'est en cela qu'elles relèvent du fonctionnement économique du psychisme et illustrent le principe d'économie de l'énergie de la pensée ${ }^{33}$.

Ces positions sont des échos de la compréhension humboldtienne du langage comme mise en forme dont la tâche consisterait à formater la substance ou le matériau langagier. Enfin, pour les tenants de la syntaxe psychologique, la relation syntaxique possède un équivalent psychique, voire psychophysique, dans la mesure où elle est posée comme un fait psychique qui possède ses corrélats sensoriels ${ }^{34}$.

\section{CONCLUSION}

L'interprétation du classement morphologique des langues en termes d'expression des états psychiques a constitué une étape significative de l'émergence du courant psychologique. En effet, depuis Bopp, la linguistique historique et comparée avait mis l'accent sur l'aspect phonique ou sonore, ou encore substantiel des langues. Ainsi, la « forme » qui a fondé cette approche a été perçue dans sa matérialité. Le courant psychologique naissant se fixe pour but de désubstantialiser la « forme » héritée de la méthode historique et comparée. En d'autres termes, le langage est conçu, pour reprendre la célèbre formule humboldtienne, non pas comme le produit d'une activité psychique (ergon), mais il est lui-même cette activité (energeia). Il s'agit désormais d'examiner le langage dans son dynamisme, dans son action, comme une activité de la parole, tributaire de dispositifs psychiques particuliers. Tel est le sens de la démarche de Steinthal qui accomplit cette désubstantialisation de la morphologie des langues dans son ouvrage consacré à leur classification (Die Classification der Sprachen) de $1850^{35}$. Cette classification ne suit pas le principe « externe » (c'est-à-dire la substance des langues), mais uniquement le principe « interne » (ou la «forme

33 Rappelons que, pour Ovsianiko-Koulikovski, le travail syntaxique s'accomplit de manière automatique « dans la sphère inconsciente près du seuil de la conscience ». Selon l'auteur, ce travail vise les formes grammaticales, il est indispensable pour que le contenu de la conscience puisse se transformer en un acte de pensée. En paraphrasant, on peut dire qu'il s'agit pour l'auteur d'une « sublimation » du contenu de la conscience, c'est-à-dire des représentations, par les éléments syntaxiques. C'est par la syntaxe que passe la sublimation des éléments matériels du psychisme, c'est-à-dire des représentations. Il s'agit de fait d'un travail formaliste qui vise à explorer les relations entre les « éléments » de la conscience, c'est-à-dire, les représentations. C'est l'ordre, l'organisation (uporjadočennost') qui conduit à la formation d'une combinaison syntaxique à partir des mots donnés. Ce travail intellectuel vise l'établissement de relations internes entre certains éléments grammaticaux ou morphologiques (voir Ovsianiko-Koulikovski, Syntaxe du russe, dans Ščeulin \& Medvedeva 1965 : 317-318).

34 En effet, selon Ovsianiko-Koulikovski, l'emploi du prédicat s'accompagne, dans la conscience du locuteur, d'une sensation particulière : la sensation prédicative (ou de la prédicativité : Oščuščenije skazuemosti ili predicirovanije). Cette sensation confère au prédicat un poids relatif plus important par rapport aux autres membres de la proposition (ibid. : 320). Pechkovski et Chakhmatov, quant à eux, insistent sur l'intervention de la volonté dans chaque combinaison syntaxique, dans la mesure où il s'agit d'assurer un assemblage de plusieurs représentations et de complexes de représentations.

35 Trautmann-Waller 2006 : 53. Voir en outre, sur le classement des langues selon leurs «formes internes », Steinthal $1860: 317-318$ et 127-130. Les trois étapes de l'évolution de la forme interne sont spécifiées, en particulier, dans Steinthal 1881 [1871] : 396-431. 
interne des langues »), donc du point de vue «énergétique» et dans une perspective psychologique.

En Russie, la "psychologisation » de la «forme» du langage s'accomplit surtout dans l'œuvre de Potebnia. Interprète de Humboldt et de Steinthal, cet auteur assimile expressément l'évolution de la langue à l'évolution de la pensée (Potebnia 1999 [1862]). L'école linguistique de Kharkov fondée par A. Potebnia est d'une importance cruciale dans le développement de la tradition psychologique russe. C'est elle qui a transféré dans les sciences russes du langage l'ensemble conceptuel du courant psychologique allemand: notion de représentation (Vorstellung) et de sentiment (Gefühl), de loi d'association, d'aperception, de " geste langagier », problématique de la «zone claire » et de la «zone obscure » de la conscience, concepts herbartiens de série, de fusion, de complexification et d' "étroitesse de la conscience», ainsi que toute une problématique ethnopsychologique (voir Bumann 1965: 60). Ce complexe conceptuel, transposé de manière plus ou moins fidèle et homogène dans le contexte russe, a été appliqué à l'analyse du langage et de la littérature par Ovsianiko-Koulikovski et d'autres disciples de Potebnia représentant ce qu'on a coutume d'appeler le « courant psychologique » russe, centré sur la «psychologie de la pensée et du sentiment» et la «psychologie de la création» (Osmakov 1981).

Le « courant néo-grammairien russe », dit aussi école linguistique de Kazan, celle de Jan Baudouin de Courtenay, et l'école linguistique de Moscou, celle de Fortounatov, ont puissamment contribué à imposer la dimension psychologique dans les sciences du langage nationales. Cette perspective psychologique, fondée sur l'idée de synchronie et le principe de l'analogie, a graduellement remplacé les approches caractéristiques du comparatisme classique et a alors pu constituer un véritable «cadre épistémologique» pour la linguistique russe à venir. Il n'est donc pas étonnant que tous les linguistes russes majeurs durant la seconde moitié $\mathrm{du} \mathrm{XIX}^{\mathrm{e}}$ siècle et le début $\mathrm{du} \mathrm{XX}^{\mathrm{e}}$ siècle s'inscrivent dans le programme de la linguistique psychologique et déclarent fermement vouloir travailler à son développement en tant que seule discipline du domaine se révélant digne, à leurs yeux, d'intérêt scientifique.

\section{BIBLIOGRAPHIE}

Bumann, Waltraud. 1965. Die Sprachtheorie Heymann Steinthals. Meisenheim: Anton Hain.

Chakhmatov, Alekseï. 2007 [1925]. Sintaksis russkogo jazyka [La syntaxe du russe]. Moscou : URSS.

Danilenko, Valeri. 2009. Istorija russkogo jazykoznanija [Histoire de la linguistique russe]. Moscou : Flinta \& Nauka.

Fortounatov, Filipp. 1956. Izbrannyje trudy [Euvres choisies]. 2 vol. Moscou : Gos. Učebno-pedagogičeskoje izd.

Gabelentz, Georg von der. 1869. Ideen zu einer vergleichenden Syntaxe. Zeitschrift für Völkerpsychologie und Sprachwissenschaft 6 : 376-384. 
Gabelentz, Georg von der. 1874. Weiteres zur vergleichenden Syntax. Wort- und Satzstellung. Zeitschrift für Völkerpsychologie und Sprachwissenschaft 8 : 300338.

Gabelentz, Georg von der. 1972 [1901]. Die Sprachwissenschaft. Ihre Aufgaben, Methoden und bisherigen Ergebnisse. Tübingen : Universität von Tübingen.

Graffi, Giorgio. 2001. 200 Years of Syntax. A Critical Survey. Amsterdam \& Philadelphie : John Benjamins.

Herbart, Johann Friedrich. 1965 [1816]. Lehrbuch zur Psychologie. Amsterdam : E. J. Bonset.

Osmakov, Nikolaj. 1981. Psixologičeskoje napravlenie v russkom literaturovedenii. D. N. Ovsjaniko-Kulikovskij [Le courant psychologique dans la critique littéraire russe. D. N. Ovsjaniko-Kulikovskij]. Moscou : Prosveščenie.

Ovsianiko-Koulikovski, Dimitri. 1912. Sintaksis russkogo jazyka [La syntaxe du russe]. Saint-Pétersbourg : izd. I. L. Ovsjaniko-Kulikovskoj.

Ovsianiko-Koulikovski, Dimitri. 1965 [1912]. Sintaksis russkogo jazyka [La syntaxe du russe], dir. par Vasilij Ščeulin \& Valerija Medvedeva. Xrestomatija po istorii grammatičeskix učenij $v$ Rossii [Anthologie de l'histoire des théories grammaticales en Russie]. Moscou : Vysšaja Škola.

Ovsianiko-Koulikovski, Dimitri. 1989. Literaturno-kritičeskije raboty v 2 tomax [Essais de critique littéraire en 2 volumes], vol. 1. Moscou : Xudožestvennaja literatura.

Paul, Hermann. 1909 [1880]. Prinzipien der Sprachgeschichte. Tübingen : Max Niemeyer.

Pechkovski, Aleksandr. 2001 [1938]. Russkij sintaksis v naučnom osveščenii [La syntaxe du russe expliquée scientifiquement]. Moscou : URSS.

Pechkovski, Aleksandr. 2010. Ob'ektivnaja i normativnaja točka zrenija na jazyk [La langue du point de vue objectif et normatif]. Moscou : URSS.

Potebnia, Aleksandr. 1958 [1874]. Iz zapisok po russkoj gramamtike [Notes sur la grammaire russe], vol. 1-2. Moscou : Gosudratsvennoje učebno-pedagogičeskoje izdatelstvo Ministrestva Prosveščenija RSFSR.

Potebnia, Aleksandr. 1968 [1899]. Iz zapisok po russkoj grammatike [Notes sur la grammaire russe], vol. 3. Moscou : Prosveščenie.

Potebnia, Aleksandr. 1970 [1905]. Iz zapisok po teorii slovesnosti [Notes philologiques]. Paris \& La Haye : Mouton.

Potebnia, Aleksandr. 1999 [1862]. Mysl'i jazyk (Pensée et langage). Moscou : Labirint [La Pensée et le langage, trad. fr. par Patrick Sériot et Margarita Schönenberger. Limoges : Lambert-Lucas (à paraitre, 2022)].

Romand, David \& Sergueï Tchougounnikov. 2009. Aux origines allemandes de la pensée cognitiviste. Introduction. Revue d'histoire des sciences humaines 21(2): 3-27.

Steinthal, Heymann. 1855. Grammatik, Logik und Psychologie. Ihre Principien und ihr Verhältniss zu einander. Berlin : F. Dümmler.

Steinthal, Heymann. 1858 [1851]. Der Ursprung der Sprache. Im Zusammenhange mit den letzten Fragen alles Wissens. Eine Darstellung, Kritik und Fortentwicklung der vorzüglichsten Ansichten. Berlin : F. Dümmler. 
Steinthal, Heymann. 1860. Charakteristik der hauptsächlichsten Typen des Sprachbaues. $2^{\mathrm{e}}$ éd. Berlin : F. Dümmler.

Steinthal, Heymann. 1881 [1871]. Abriss der Sprachwissenschaft, B. 1 : Die Sprache im Allgemeinen. Einleitung in die Psychologie und Sprachwissenschaft. $2^{\mathrm{e}}$ éd. Berlin : F. Dümmler \& Harrwitz und Gossmann.

Ščeulin, Vasilij \& Valerija Medvedeva. 1965. Xrestomatija po istorii grammatičeskix učenij $v$ Rossii [Anthologie de l'histoire des théories grammaticales en Russie]. Moscou : Vysšaja Škola.

Trautmann-Waller, Céline. 2006. Aux origines d'une science allemande de la culture. Linguistique et psychologie des peuples chez Heymann Steinthal. Paris : CNRS Éditions.

Vygotski, Lev. 1996 [1934]. Myšlenije i reč [Pensée et langage]. Moscou : Labirint.

Wundt, Wilhelm. 1900. Völkerpsychologie. Eine Untersuchung der Entwicklungsgesetze von Sprache, Mythus und Sitte. 2 vol. Leipzig : W. Engelmann.

Wundt, Wilhelm. 2002 [1902-1914]. Psixologija narodov [La psychologie des peuples]. Moscou : Eksmo. 
